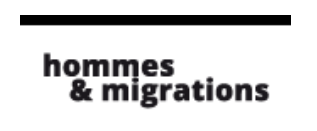

Hommes \& migrations

Revue française de référence sur les dynamiques

migratoires

$1301 \mid 2013$

Migrations et mondes ruraux

\title{
Quand les saisonniers tunisiens de Ghardimaou interrogent le codéveloppement
}

\section{Swanie Potot}

\section{(apenEdition \\ Journals}

\section{Édition électronique}

URL : http://journals.openedition.org/hommesmigrations/1919

DOI : 10.4000/hommesmigrations.1919

ISSN : 2262-3353

\section{Éditeur}

Musée national de l'histoire de l'immigration

\section{Édition imprimée}

Date de publication : 1 janvier 2013

Pagination : 79-87

ISBN : 978-2-919040-21-6

ISSN : $1142-852 X$

\section{Référence électronique}

Swanie Potot, "Quand les saisonniers tunisiens de Ghardimaou interrogent le codéveloppement », Hommes \& migrations [En ligne], 1301 | 2013, mis en ligne le 31 décembre 2015, consulté le 01 mai 2019. URL : http://journals.openedition.org/hommesmigrations/1919; DOI : 10.4000/ hommesmigrations.1919 


\title{
QUAND LES SAISONNIERS TUNISIENS DE GHARDIMAOU INTERROGENT LE CODÉVELOPPEMENT
}

par SWANIE POTOT, sociologue, chargée de recherche au CNRS,

unité de recherche Migrations et Société (URMIS), université de Nice-Sophia-Antipolis.

\author{
Depuis les années 1970, les Tunisiens de la région \\ de Ghardimaou migrent de façon temporaire vers la vallée \\ du Rhône pour subvenir aux besoins de leurs familles restées \\ au pays. La migration pendulaire fondée sur le retour du migrant \\ après la période de travail n'est donc pas un phénomène \\ nouveau. Elle connaît pourtant un regain d'intérêt de la part \\ des autorités européennes. Dans un contexte qui rend la \\ circulation migratoire de plus en plus difficile, cette conception \\ d'un partenariat gagnant-gagnant a tout lieu de se changer \\ en jeu de dupes.
}

\section{II s'agit de présenter les débuts de cette migration vers la France dans les années 1970 et le modèle social et familial qu'elle a engendré à Ghardimaou.}

Une chaîne migratoire s'est développée à partir des années 1970 entre un petit village tunisien, Ghardimaou, et la basse vallée du Rhône. Cette contribution rend compte du rôle de ces travailleurs dans l'économie française agricole et aborde la notion de codéveloppement en interrogeant les conséquences sociales et économiques de cette migration sur le tissu social d'origine. Il s'agit de présenter les débuts de cette migration vers la France dans les années 1970 et le modèle social et familial qu'elle a engendré à Ghardimaou. Dans un deuxième temps, est analysée la création d'une niche spécifique à ces migrants, dans le secteur maraîcher rhodanien où ils ont accompagné la restruc- turation des exploitations agricoles. La perspective diachronique souligne que les migrations actuelles, notamment celles qui s'effectuent à grands risques en traversant illégalement la Méditerranée, ne sont pas novatrices, elles s'inscrivent dans une tradition de mobilité ancienne, aujourd'hui perturbée par des politiques migratoires restrictives.

Depuis les années 2000, nombreux sont les États européens (Espagne, Italie, Allemagne, Autriche, Grande-Bretagne...) qui ont mis en place ou renouvelé des accords de migrations temporaires avec des pays tiers dans le but d'importer la main-d'œuvre nécessaire à l'économie occidentale sans s'engager à assurer les conditions d'une immigration à 
long terme ${ }^{1}$. Promus par l'Union européenne, les contrats proposés, cantonnés à quelques secteurs économiques - en premier lieu l'agriculture - et limités par quotas, permettent de recruter des salariés pour quelques mois, tandis qu'ils sont encore dans leur pays d'origine, en les obligeant à y retourner à la fin de la saison s'ils veulent pouvoir postuler à nouveau à ce dispositif l'année suivante.

\section{Les migrations saisonnières, un accord gagnant-gagnant ?}

Les instances européennes et les organisations internationales telles que l'Office international des migrations (OIM) mettent en avant le "partenariat gagnant-gagnant" de ces migrations saisonnières très encadrées : elles

Promus par l'Union

européenne, les contrats

proposés, cantonnés

à quelques secteurs

économiques - en premier

lieu l'agriculture - et limités

par quotas, permettent

de recruter des salariés

pour quelques mois, tandis

qu'ils sont encore

dans leur pays d'origine,

en les obligeant à y retourner

à la fin de la saison. forme aboutie de codéveloppement, le "Nord" soutenant, via les salaires des travailleurs saisonniers, des investissements dans les pays du "Sud", tout en dynamisant sa propre économie ${ }^{2}$.

La France, terre d'immigration ancienne, fut précurseur dans le domaine avec la mise en place de telles mesures dès l'après-guerre ${ }^{3}$. Le dispositif fut d'abord réservé aux ressortissants européens avant de s'ouvrir, dans les années 1960, aux Marocains ${ }^{4}$ et aux Tunisiens ${ }^{5}$, ces deux nationalités formant aujourd'hui la grande majorité des saisonniers étrangers employés dans le maraîchage français. C'est donc le but d'évaluer les effets sur le long terme d'un dispositif récemment remis au goût du jour à l'échelle européenne que nous avons choisi de nous intéresser aux travailleurs tunisiens dans l'agriculture française. L'hypothèse du partenariat gagnantgagnant nous invitait à enquêter des deux côtés de la Méditerranée, de façon à comprendre, d'une part, le contexte socio-économique dans lequel s'insère le travail de ces ouvriers en France et, d'autre part, de saisir les conséquences sociales et économiques de ces migrations sur leur région d'origine.

Les conclusions présentées ici s'appuient sur deux programmes de recherche. Le premier portait sur le travail des étrangers dans l'agriculture marâ̂chère du sud de la France et fut conduit par une équipe de 7 chercheurs entre 2006 et 2009 ; le deuxième, centré sur les migrations tunisiennes vers l'Europe, a été mené par une équipe de 9 universitaires en Tunisie entre 2010 et $2012^{6}$.

Dans les deux cas, nous avons enquêté auprès des multiples acteurs impliqués de près ou de loin dans ces migrations : migrants, employeurs, syndicalistes et associations de défense des travailleurs étrangers, familles de migrants, fonctionnaires de l'Inspection du travail agricole (ITEPSA), de l'Office français de l'immigration et de l'intégration (OFII ex-ANAEM et antérieurement OMI), de l'Office des Tunisiens de l'étranger (OTE), d'administrations locales tunisiennes (délégués et chefs de secteurs), etc. Au cours de ces six années, environ 200 entretiens ont été recueillis et enrichis par les statistiques et les informations officielles disponibles sur le sujet tant en France qu'en Tunisie. C'est une partie des données de chacun de ces pro-

\footnotetext{
1. Alain Morice, "Le travail sans le travailleur", in Plein droit, 2004, pp. 2-7; Olivier Clochard, Réseau migreurop (dir.), Atlas des migrants en Europe. Géographie critique des politiques migratoires, Paris, Armand Colin, 2009. 2. Philippe Courtin, "Le codéveloppement : un alibi pour des politiques migratoires restrictives", in Revue internationale et stratégique, $\mathrm{n}^{\circ}$ 4, 2007, pp. 43-47. 3. Ordonnance $\mathrm{n}^{\circ}$ 452658 du 2 novembre 1945 relative aux conditions d'entrée et de séjour en France des étrangers et portant création de l'Office national

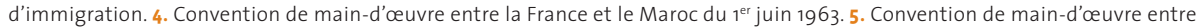
la France et la Tunisie du 9 août 1963, reprise dans le cadre de l'Accord cadre relatif à la gestion des flux concertés et au développement solidaire signé entre la France et la Tunisie en 2008. 6. En France, il s'agit du programme ANR-JCJC Migragri, dirigé par l'auteure ; en Tunisie, il s'agit du programme IRD-université de Sousse Micodev, dirigé par H. Boubakri et l'auteure.
} 
grammes qui est exploitée dans le présent article. En France, au début des années 2000, on constatait que plus de la moitié des contrats saisonniers gérés par l'Office des migrations internationales (OMI) chaque année ${ }^{7}$ étaient destinés au département des Bouches-du-Rhône, suivi du Rhône, du Vaucluse, du Gard et de la Drôme ${ }^{8}$. C'est pourquoi la basse vallée du Rhône a été choisie comme lieu d'enquête. À partir du terrain français, nous avons rapidement découvert que le millier de Tunisiens concernés annuellement par ces contrats, comme leurs compatriotes employés à l'année ou illégalement à leurs côtés, étaient presque tous originaires du gouvernorat de Jendouba, dans le nord-ouest de la Tunisie, et plus spécifiquement rattachés à la délégation de Ghardimaou ${ }^{9}$, une entité administrative de 68000 habitants, frontalière de l'Algérie, où nous avons effectué six séjours d'enquête ${ }^{10}$. Des deux côtés de la Méditerranée, si les migrations saisonnières étaient au cœur de la recherche, nous nous sommes également intéressés à d'autres modalités migratoires (longs séjours, migrations illégales, migrations de travailleurs détachés, etc.) et à des migrations originaires d'autres régions et pays afin d’appréhender le phénomène dans son contexte et de comprendre en quoi il est orienté par - et influence lui-même - d'autres mobilités.

\section{Une mobilité ancienne}

Il faut remonter aux années 1960 et 1970 pour comprendre comment s'est formée la chaîne migratoire de Ghardimaou vers le sud de la France. À cette époque, le gouvernorat de Jendouba, éloigné de la capitale et relativement délaissé par les pouvoirs publics, offrait peu de ressources. Étant donné son relief escarpé, les programmes de développement agricole mis en œuvre après la décolonisation se sont arrêtés aux portes de Ghardimaou. La principale ressource du village était liée à sa position stratégique proche de l'Algérie. Une partie des hommes y vendaient alors leur force de travail plusieurs mois dans l'année. Parallèlement, les montagnes de la Kroumirie, qui forment la frontière au niveau du village, ont toujours facilité les trafics de marchandises de toutes sortes, revendues ensuite à des compatriotes

De ce fait, il semble que les migrants originaires d'autres régions, ce qui permettait - et permet encore de Ghardimaou étaient moins concentrés sur le territoire français qu'ils ne le sont aujourd'hui. nombre d'habitants de ne pas tomber dans la pauvreté. Les avantages de la migration transfrontalière, qu'elle soit commerciale ou de travail, étaient donc exploités de longue date. Jusqu'à la fin des années 1960, les migrations vers l'ancien pays colonisateur se pratiquaient également mais de façon marginale. C'est seulement à partir des années 1970 qu'elles devinrent une source d'enrichissement notable pour les habitants de la région.

“À l'époque cétait facile. Le plus dur, cétait d'acheter le billet de bateau. C'était 300 francs. Après t'allais à La Goulette [port de Tunis], tu prenais le bateau et à Marseille tu cherchais du travail. Voilà, cétait aussi compliqué que ça ${ }^{11}$."

Les témoignages recueillis auprès de personnes aujourd'hui retraitées édulcorent probablement les difficultés rencontrées lors de ces mobilités, mais ils témoignent au moins d'une grande liberté de circulation. Non seulement la frontière entre la Tunisie et la France était relativement ouverte ${ }^{12}$, mais les opportunités de travail étaient plus nombreuses. De ce fait, il semble qu'alors les migrants originaires de Ghardimaou étaient moins concentrés sur le territoire français qu'ils ne le sont aujourd'hui. Beaucoup résidaient quelque temps dans la région de Marseille, soit parce qu'ils y avaient des liens, soit simplement parce qu'ils y débarquaient, mais les déplacements vers d'autres départements français étaient importants. Tout le sud de la France a ain- 
si bénéficié de manœuvres en maçonnerie venus de Ghardimaou, tandis que certains trouvaient à se faire employer dans l'industrie, notamment chez Peugeot à Sochaux, et que d'autres partaient explorer des régions de faible immigration comme la Bretagne ou la Normandie. L'agriculture du sud de la France ne représentait alors qu'une modalité de travail parmi d'autres. Chez ceux qui s'orientaient vers ce secteur, il semble que les contrats saisonniers gérés par l'OMI n'étaient que très rarement contractés comme il se devait, depuis le pays d'origine. Comme pour les autres titres de séjour, c'est plutôt en France que la situation était régularisée a posteriori. Dès cette époque, les migrations vers la France sont devenues une composante de la vie sociale de cette région. Les jeunes hommes auxquels la famille n'offrait pas de capital économique envisageaient aisément de partir dès la fin de l'adolescence pour s'assurer une situation et se donner les moyens de contracter un mariage. La migration, en elle-même, faisait alors office d'emploi sûr et bien rémunéré auprès des familles des jeunes femmes qui n'hésitaient pas à marier leur fille à un "immigré13".

D'après les témoignages recueillis, même s'il était à l'époque plus facile qu'aujourd'hui de s'installer durablement en France, la migration n'était nullement le symbole d'une rupture avec le pays d'origine. Au contraire, elle permettait de fonder un foyer à Ghardimaou, la plupart du temps avec une femme issue du même village, voire de sa propre famille ${ }^{14}$. Après cette alliance, les épouses rejoignaient parfois - rarement - leur mari en France durant quelques mois ou années. Toutefois, dès que les premiers enfants atteignaient l'âge d'être scolarisés, la norme consistait à les élever en Tunisie, avec leur mère, tandis que le père ne revenait que quelques semaines par an. S'il s'agissait d'une migration essentiellement masculine, les femmes y jouaient un rôle structurant en assurant le développement de familles transnationales ; elles étaient des relais stables entre le migrant et sa terre d'origine. Malgré la distance, les migrants ne cessaient de communiquer avec leur femme, donnant des instructions quant à l'éducation des enfants ou à la gestion du capital, et ils étaient en retour tenus au courant de la vie du village. Ce sont ces femmes qui confirmaient la présence sociale du migrant sur place durant ses séjours à l'étranger. Mais cette coprésence n'était possible que parce qu'elle était accompagnée de la liberté de circuler : celle-ci assurait non seulement des retours réguliers, mais elle contribuait à diminuer la distance dans les esprits, autorisant le migrant à rentrer à tout moment, en cas de nécessité. En ce sens, la liberté de circulation qui a prévalu jusqu'en 1986 diminuait l'attractivité du regroupement familial.

Les salaires venus de France permettaient de subvenir aux besoins de la famille en termes d'alimentation, de soins, d'habillement et d'habitat (rénovation, amélioration de la maison) et autorisaient souvent la constitution d'un petit capital investi au village plus tard, au moment de la retraite, dans la construction d'une villa et, plus rarement, dans le montage d'un commerce ou l'achat de matériel agricole pour l'exploitation du domaine familial. Il est indéniable que ces mouvements apportaient dans cette délégation relativement démunie un afflux financier conséquent et contribuaient à améliorer le niveau de vie de ses habitants. Ce faisant, les migrants sont devenus une composante de plus en plus indispensable d'une société dans laquelle ils faisaient figure de golden boys.

\section{L'agriculture comme niche face aux restrictions migratoires}

Dans les années 1980 et 1990, la relative fermeture des frontières a conduit les migrants à concentrer leur activité migratoire dans l'agriculture de la basse vallée du Rhône, et marginalement en Corse, où leur présence était encore tolérée car elle compensait les effets de la "libéralisation" de l'agriculture européenne. Si aller en France était encore possible pour le plus grand nombre au début des années 1980, cela 
Une hiérarchie s'opère au sein même du "bidonville". Les plus anciens vivent souvent dans des caravanes et les nouveaux arrivants sous des bâches, des cahutes improvisées. Mais ces lieux restent des lieux de vie, même s'ils sont des territoires de précarité. @ Yohanne Lamoulére / Transit / Picturetank

est devenu problématique à partir de l'introduction des visas en 1986 et plus encore durant les décennies suivantes avec la mise en œuvre de l'espace Schengen, la restriction progressive de la délivrance des titres de séjour et, à partir des années 2000, la lutte contre le travail dissimulé. Pour les nouveaux candidats au départ, souvent eux-mêmes issus de familles migrantes, partir est alors devenu une épreuve.

Ces restrictions ont eu pour effet de canaliser la migration de Ghardimaou. Jusqu'alors, beaucoup étaient partis à l'aventure vers la France en espérant se débrouiller sur place mais, avec la fermeture des frontières, le capital social propre à la migration est devenu de plus en plus nécessaire ${ }^{15}$. C'est souvent grâce à l'aide de parents partis avant eux que les nouveaux migrants parvenaient à se faire embaucher dans des niches réduites, comme le bâtiment et l'agriculture, restées encore ouvertes aux étrangers dont la situation était de plus en plus souvent irrégulière. Cette nouvelle donne a favorisé le départ des proches de migrants et restreint les possibilités de ceux qui n'avaient pas de relations à l'étranger. Même dans le village de Ghardimaou, fortement touché par la migration, certaines familles se sont trouvées dans l'impossibilité de partir car aucun de leurs membres n'avait choisi cette option avant les mesures de restrictions. Dans un tel contexte, les contrats de saisonnier agricole, 
qui étaient dans la période précédente une modalité parmi d'autres, sont devenus une voie d'accès privilégiée.

En France, les producteurs de fruits et légumes se sont mobilisés de longue date pour conserver l'autorisation d'embaucher de la main-d'œuvre étrangère, arguant de la spécificité du secteur. Celui-ci a, en effet, été très affecté par la "mondialisation" de l'économie : le développement des transports et la libre concurrence internationale, cumulés à la modernisation technologique et aux nouveaux modes de distribution commerciale, ont considérablement réduit les marges de profit des agriculteurs. À partir de la fin des années 1980, la production française s'est profondément transformée pour tenter de se maintenir sur un marché très concurrentiel. Un des éléments sur lesquels la profession a choisi de jouer est le coût du travail salarié et la flexibilité de sa gestion.

La nécessité de pouvoir recruter et débaucher rapidement des contingents importants d'ouvriers en fonction non seulement des cycles de production mais également des aléas

Ce faisant, les migrants sont

devenus une composante

de plus en plus indispensable

d'une société dans

laquelle ils faisaient figure

de golden boys. météorologiques et des fluctuations de la demande de la grande distribution conduit à favoriser le recrutement temporaire d'étrangers ${ }^{16}$. Ceux-ci constituent une armée de réserve illimitée, mobilisable à tout moment, prête à accepter de maigres salaires - mais élevés comparativement à ceux perçus dans leur pays d'origine - et à adapter leurs horaires à la quantité de travail. Afin de répondre à ces exigences, les accords spécifiques d'introduction de travailleurs saisonniers ont été maintenus dans l'agriculture maraîchère malgré les restrictions im- posées à l'immigration de travail. Les abus occasionnés par la position très dépendante et dominée de ces travailleurs ont été dénoncés maintes fois ${ }^{17}$, mais ces contrats n'en restent pas moins très prisés de la part de migrants volontaires dont les opportunités pour partir vers l'Europe sont minimes. Depuis la fin des années 1990, ils représentent l'unique moyen de se maintenir dans une circulation pendulaire, passant six à huit mois par an en France, le reste en Tunisie. Ce dispositif permet aux nouvelles générations de vivre la migration dans une perspective transnationale, comme l'avaient pratiquée leurs pères. L'interdiction faite à ces saisonniers de regrouper leur famille en France n'est pas vécue comme une contrainte mais comme la prolongation d'une norme dont ils ont eux-mêmes fait l'expérience dans leur enfance.

La forte attractivité de cette modalité migratoire séduit encore aujourd'hui de nombreux candidats, mais le peu de contrats offerts aux Tunisiens, entre 500 et 1000 par an depuis 1990, en a impliqué une distribution parcimonieuse. Contrairement à ce qui a été observé par d'autres au Maroc ${ }^{18}$, ici, le petit nombre de places disponibles chaque année pour cause de départ en retraite ou de rupture entre un salarié et son employeur ne fait pas systématiquement l'objet de transactions pécuniaires ; ce sont essentiellement les enfants de saisonniers déjà embauchés qui peuvent en bénéficier, avec l'accord parfois monnayé - des employeurs français.

Malgré des quotas apparemment limités, ce système de contrats saisonniers a développé une niche migratoire majeure pour la délégation de Ghardimaou au cours des vingt dernières années. D'une part, certains anciens saisonniers ont changé de statut. Les plus chanceux sont parvenus à obtenir un titre de séjour de longue durée, souvent

16. Jean-Pierre Berlan, “Dynamique d'intégration dans l'agriculture provençale”, in Études rurales, 1994, pp. 151-16o ; Françoise Bourquelo, Salariat agricole et migrations en Méditerranée, Solagral, 1996 ; Béatrice Mésini, “Flexi-insécurité dans un secteur en tension : processus de segmentation statutaire et ethnique du marché des saisonniers agricoles", in Asylon(s) - La revue des deux asiles, 2008. 17. Frédéric Decosse, “Le nouveau serf, son corps et nos fruits et légumes", in Plein droit, 2008 ; "Travailleurs saisonniers dans l'agriculture européenne”, in Études rurales, n 182, EHESS, 2008 ; Alain Morice, “Pas de séjour sans travail, ou les pièges du contrat saisonnier. L'exemple des Marocains dans l'agriculture provençale”, in Migrations société, n 18, 2006, pp. 211-231 ; Swanie Potot, “La précarité sous toutes ses formes : concurrence entre travailleurs étrangers dans l'agriculture française”, in Alain Morice, Swanie Potot (dir.) De l'ouvrier sans papiers au travailleur détaché. Les migrants dans la modernisation du salariat, Paris, Karthala, 2010, pp. 201-224. 18. Alain Morice, "Pas de séjour sans travail, ou les pièges du contrat saisonnier. L'exemple des Marocains dans l'agriculture provençale", art. cit. 
à la demande de leur employeur dans le but d'en faire des travailleurs permanents. Ils sont alors promus chefs d'équipe ou responsables d'exploitation, ce qui libère du même coup un poste de saisonnier et place ce salarié en position de favoriser le recrutement de compatriotes à l'avenir.

\section{La condition précaire des saisonniers sans papiers}

Des saisonniers quittent parfois leur poste pour rester sur le sol français sans titre de séjour. C'est là un autre effet de la fermeture des frontières et de la précarité du statut : le renouvellement du contrat d'année en année ne va pas de soi, l'employeur conserve la possibilité de ne pas reprendre un saisonnier, notamment si celui-ci ne s'est pas montré suffisamment complaisant ou endurant durant la saison antérieure. Le migrant rentré au village perd alors toute opportunité de repartir puisque, depuis la Tunisie, il aura toutes les difficultés à obtenir un nouveau titre de séjour pour la France. C'est alors le mode de vie de toute une famille qui s'effondre.

En 2005, suite au déclenchement d'une grève des saisonniers pour réclamer le paiement d'heures supplémentaires dans une grande exploitation, plus d'une centaine de migrants ont appris après leur retour au pays qu'ils ne seraient pas reconduits dans leur emploi malgré les promesses qui leur avaient été faites. Raouf, rencontré à Ghardimaou, faisait partie de ceux-là : "J'ai travaillé de 2000 à 2005 dans la plaine de la Crau, mais après c'est fini. C'est à cause du préfet, il a promis qu'on serait réembauchés en priorité. Mais cétait pas vrai, les autres patrons ne voulaient plus de nous, parce qu'on était vu comme des fouteurs de m... Ils ont attendu qu'on rentre, tranquillement, et après ils nous ont laissés là, comme des chiens. Moi j'ai plus rien. En saisonnier, tu gagnes pas assez pour mettre [de l'argent] de côté. [...] Après, j'ai plus trouvé de travail ici. Chômeur, deux ans. Main- tenant je travaille dans la plantation de tabac mais je gagne presque rien. J'habite dans une cabane là-bas, dans la plantation. C'est la misère ${ }^{19}$."

Pour éviter de se trouver un jour dans une telle situation, certains font le choix de ne pas repartir à la fin d'une saison. Ils restent alors sans titre de séjour, dans l'espoir de travailler au noir et d'être régularisés. Quelques-uns parviennent à contracter un mariage et d'autres, peu nombreux, ont obtenu un titre de séjour après dix ou quinze ans de clandestinité sur le territoire français ${ }^{20}$. Mais la majorité d'entre eux sont astreints à la clandestinité pour de longues années, intervenant ponctuellement comme main-d'œuvre surnuméraire dans l'agriculture
Malgré des quotas apparemment limités, ce système de contrats saisonniers a développé une niche migratoire majeure pour la délégation de Ghardimaou au cours des vingt dernières années. locale, vivant très chichement et se logeant dans quelques "bidonchamps" près d'exploitations agricoles où ils sont tolérés ${ }^{21}$. D’après les témoignages recueillis, plusieurs centaines de personnes issues de la délégation de Ghardimaou seraient aujourd'hui dans cette situation, principalement dans les Bouches-du-Rhône, la Drôme et le Vaucluse.

Ces clandestins sont parfois rejoints par des migrants qui, faute de trouver mieux, sont partis vers la France pour y séjourner illégalement. Souvent, ils achètent au prix fort un contrat de travail auprès de trafiquants qui s'entendent avec des employeurs français ou italiens pour établir de fausses promesses d'embauche, dans le seul but d'obtenir un visa de travail. Une petite partie des contrats saisonniers, surtout ceux orientés vers la Corse, ont été explicitement détournés à cette fin. Une fois sur place, le migrant ne se rend pas auprès de l'employeur mentionné mais tente de mettre à profit ses connaissances pour trouver un emploi, un logement, etc. Ici encore, la possession d'un réseau social sur place fait toute la différence. On distingue notamment le cas des fils d'anciens migrants soute- 
nus dans leur expédition par leur père. Ils sont pris en charge par des proches et parviennent souvent à éviter l'agriculture en travaillant informellement dans de petites entreprises du bâtiment tenues par des amis en règle. En revanche, lorsque les nouveaux arrivants ne peuvent compter sur le soutien de proches, ils se rendent à Berre-l'Étang ou dans le Vaucluse pour tenter de travailler dans l'agriculture. C'est notamment cette option qui est choisie par la plupart des migrants les plus démunis : ils traversent la Méditerranée au pé-
Plus que de les empêcher d'entrer, les barrières que l'Union européenne

érige à ses frontières

limitent la mobilité

des migrants et tendent à

les isoler socialement. ril de leur vie ${ }^{22}$ contre 1000 à $2000 €$, sans idée claire de ce qu'ils entreprendront une fois sur place ${ }^{23}$. Si les départs de ce type se sont intensifiés en 2011, cette pratique sobserve dans la délégation de Ghardimaou, pourtant éloignée des côtes, depuis une dizaine d'années. Pour tous ces migrants, le monde rural de la basse vallée du Rhône est perçu comme un point de chute par défaut puisqu'ils savent qu'il est déjà "domestiqué" par des compatriotes de leur village. S'ils parviennent à traverser la Méditerranée, ceux qui n'ont pas de contrats saisonniers ne peuvent en revanche faire perdurer un mode de vie basé sur la circulation. Sans papiers et avec de très faibles ressources, il leur est difficile de maintenir un lien fort avec l'autre rive et quasiment impossible de "s'intégrer" à la société française. Pour ceux qui partent en célibataires, le mariage désiré - qui est parfois la cause du départ - est perpétuellement repoussé dans l'attente d'une évolution de la situation, tandis que ceux qui ont déjà constitué une famille vivent douloureusement des séparations de plusieurs années. Plus que de les empêcher d'entrer, les barrières que l'Union européenne érige à ses frontières limitent la mobilité des migrants et tendent à les isoler socialement.

\section{Le développement de la dépendance}

Malgré la diminution sensible des profits engendrés aujourd'hui par le travail en Europe et le nombre d'obstacles et d'humiliations subis par les migrants, les sirènes de la migration font toujours recette. Bien sûr, comme le soulignait déjà Abdelmalek Sayad dans les années 1970, l'enrichissement ostentatoire des migrants dans leur village d'origine séduit encore la jeunesse ${ }^{24}$. L'exemple, certes exceptionnel, d'un migrant parti illégalement huit ans plus tôt et déjà propriétaire d'une belle villa illustre aux yeux de tous que l'eldorado raconté par l'ancienne génération est encore à portée de main, malgré les multiples échecs. Plus prosaïquement, c'est le manque d'opportunités et surtout l'absence de modèles de réussite alternatifs qui incitent les jeunes à envisager cette perspective. S'il existe une "classe moyenne" dans le territoire étudié, elle s'est constituée par la migration et en reste directement dépendante. Entre 2007 et 2010, ce sont quelque 21,7 millions de dinars (11 millions d'euros) qui ont été transférés depuis l'étranger dans la seule délégation de Ghardimaou, soit en moyenne 319 dinars par habitant contre une moyenne nationale de 212 dinars ${ }^{25}$. Les salaires gagnés en France ont permis à une partie de la population de vivre relativement confortablement malgré le sous-développement de la région. Mais ils n'ont pas impulsé de dynamique économique autonome.

Léconomie locale, notamment les secteurs du bâtiment et de l'ameublement, est certes soutenue par les projets de construction des migrants, assurant par là une certaine redistribution, mais ces transferts n'ont pas engendré de grands projets, supports d'emplois et de développement local. Les 
quelques magasins, boulangeries, drogueries ou épiceries ouverts grâce aux transferts des revenus de l'étranger apportent une ressource continue à quelques membres de familles de migrants, mais ils ne se développent guère et ont parfois besoin d'apports complémentaires pour se maintenir.

D'après de nombreux informateurs, l'environnement structurel et institutionnel porte une grande part de responsabilités dans cette absence de développement. La centralisation extrême du pouvoir sous l'ancienne dictature ainsi que le haut niveau de corruption limitaient les initiatives individuelles et n'incitaient pas à la prise de risque. Ainsi, Ali a travaillé quarante-deux ans dans l'agriculture française avant de prendre sa retraite au village. En 2004, il a investi 18000 euros pour obtenir un visa et un emploi régulier dans une entreprise de jardin à Fréjus pour son fils aîné : "Si tu mets tes économies dans le départ de quelqu'un, un fils ou n'importe qui, tu sais que cet argent va servir, qu'il va rapporter, il va leur permettre de faire quelque chose, de donner à leur femme, pour leurs enfants. Si tu le mets dans une boîte ici, tu sais que ton argent va finir dans les poches d'untel ou d'untel et ton projet, il a même pas commencé, il a déjà disparu. C'était comme ça, à ce momentlà. Maintenant [avec la révolution] on va voir si ça va changer... Moi, aujourd'hui, j'essaye de réunir l'argent pour envoyer mon autre fils, parce que j'ai plus le temps d'attendre ${ }^{26 . "}$

Il semble qu'une part non négligeable de l'argent ramené des campagnes françaises soit directement investi dans la vaste économie de la migration. En ce sens, l'exemple de Ghardimaou ne confirme pas la théorie du codéveloppement : la migration temporaire n'impulse pas ici un développement local durable, elle tend au contraire à installer dans la durée un système de dépendance envers un dispositif précaire. Or l'article de Béatrice Mésini dans ce numéro montre que le système de recrutement des ouvriers agricoles temporaires est aujourd'hui soumis à des évolutions notables lesquelles risquent de laisser les saisonniers traditionnels sur le côté. 